\title{
Las necesidades de salud y la respuesta social en una localidad rural: Metáforas y dilemas frente al consumo de alcohol
}

\author{
Mónica Carrasco Gómez, ${ }^{1}$ Guillermina Natera Rey, ${ }^{2}$ Luz Arenas Monreal, ${ }^{1}$ \\ Hortensia Reyes-Morales,' Lilian Erendira Pacheco Magaña'
}

Artículo original

\section{ABSTRACT}

\section{Background}

Harmful alcohol use is identified as a public health problem and the success of the health system response to needs will depend on the programs, the actors who implement them and their degree of acceptability among the recipient population.

\section{Objective}

To determine the perception of how political, civil, commercial and health system actors meet the health needs derived from harmful alcohol use in a rural area, with the aim of providing information for decision-making in health policies to cope with this problem.

\section{Method}

Case study with a qualitative approach, conducted in a rural town of Morelos, in which its inhabitants identified the fact that alcohol posed a health problem.

\section{Results}

Discourse is analyzed by identifying the metaphors used to make sense of this phenomenon and the dilemmas faced.

\section{Discussion and conclusion}

The authors discuss the differences in the way it is perceived and how they prevent the implementation of actions to prevent and deal with alcohol abuse and sales regulations. They conclude that designing public policies that respond to the health needs in this area requires taking into account the dilemmatic nature of the social thinking of the individuals involved in this response, which goes beyond health service provision, and incorporating social determinants (economic, political and cultural).

Key words: Health needs, alcohol abuse, community health planning, dilemmas and metaphors.

\section{RESUMEN}

\section{Antecedentes}

El consumo nocivo de alcohol se identifica como un problema de salud pública. El éxito de la respuesta del sistema de salud a las necesidades depende de los programas, los actores que los ejecutan y del grado de aceptabilidad de la población receptora.

\section{Objetivo}

Conocer la percepción sobre cómo enfrentan los actores políticos, civiles, mercantiles y del sistema de salud las necesidades de salud derivadas del consumo nocivo de alcohol en una zona rural, con la intención de brindar información para la toma de decisiones en políticas de salud dirigidas a la atención de este problema.

\section{Metodo}

Estudio de caso con enfoque cualitativo, realizado en una localidad rural de Morelos, en la cual sus habitantes identificaron que el consumo de alcohol representaba un problema de salud.

\section{Resultados}

Se analiza el discurso y se identifican las metáforas que utiliza la población para darle sentido a este fenómeno y los dilemas que enfrentan.

\section{Discusión y conclusión}

Se discuten las diferencias en la manera en que se percibe el consumo de alcohol y cómo éstas dificultan la implementación de acciones de prevención y atención del abuso del mismo así como la regulación de la venta. Se concluye que para el diseño de políticas públicas que respondan a las necesidades de salud en este tema se requiere tomar en cuenta la naturaleza dilemática del pensamiento social de los individuos que intervienen en dicha respuesta, la cual debe rebasar la prestación de los servicios de salud e incorporar los determinantes sociales (económicos, políticos y culturales).

Palabras clave: Necesidades de salud, abuso de alcohol, planificación de la salud de la comunidad, dilemas y metáforas.

Centro de Investigación en Sistemas de Salud. Instituto Nacional de Salud Pública

2 Dirección de Investigaciones Epidemiológicas y Psicosociales Instituto Nacional de Psiquiatría Ramón de la Fuente Muñiz.

Correspondencia: Dra. Mónica Carrasco Gómez. Nogales 31, Rincón Arboledas, 72460, Puebla, Pue., México. E-mail: lazulblues@hotmail.com

Recibido primera versión: 20 de agosto de 2013. Segunda versión: 10 de junio de 2014. Aceptado: 15 de octubre de 2014. 


\section{ANTECEDENTES}

El consumo nocivo de alcohol* ${ }^{*}$ se identifica en la actualidad como un problema de salud pública, ya que está considerado como el tercer factor de riesgo más importante de muerte prematura e incapacidad. ${ }^{1}$ En los jóvenes puede poner en peligro su integridad biológica y psíquica debido a los daños en el sistema neurológico que aún está en proceso de maduración..$^{2-4} \mathrm{El}$ alcohol se encuentra asociado a diversas causas de enfermedad, lesiones, accidentes y violencia; repercute en el bienestar humano y afecta a las personas, familias y comunidades, contribuyendo a la desigualdad social y sanitaria por las pérdidas económicas que ocasiona. ${ }^{1}$

En el ámbito de los sistemas de salud, el trabajo con las poblaciones se debería generar idealmente a partir de las necesidades de salud. Para la comprensión de los mismos, se han propuesto diversas clasificaciones que diferencian las necesidades sentidas, corporativas, ${ }^{5}$ normativas, comparativas y de atención. ${ }^{6}$ En su definición, se utilizan tanto indicadores epidemiológicos como metodologías que exploran la percepción de los actores involucrados. Por tanto, su conocimiento podría brindar información sobre cómo los actores enfrentan estas situaciones y así facilitar al sistema de salud el poder brindar una respuesta integral. Este estudio aborda un problema de salud relevante para la sociedad, como es el consumo nocivo de alcohol. En México existe un patrón de consumo típico de grandes cantidades por ocasión y, como fenómeno reciente, la ingesta excesiva de alcohol a edades cada vez más tempranas, la cual incluye también a las mujeres. ${ }^{7,8}$ Este patrón contribuye al incremento de problemas sociales y de salud, lo cual se hace evidente al comparar los datos con otros países cuya forma de consumo no es excesiva y por lo general la ingesta de alcohol se acompaña con alimentos. ${ }^{9,10}$

En las zonas rurales de México, donde vive cerca del 23\% de la población, ${ }^{11}$ se ha documentado que el porcentaje de la dependencia al consumo de alcohol es mayor que en las urbanas $(10.5 \%$ vs. $9.3 \%) .^{12}$ Se ha reportado que en estas regiones las personas no identifican a este problema como una necesidad de salud debido a la aceptación social. ${ }^{13}$ Por consiguiente, difícilmente se presenta como una demanda de consulta hasta que aparece una comorbilidad o secuela social grave. ${ }^{14}$

Las consecuencias a la salud en este contexto pueden agravarse por las condiciones de precariedad de la población y del poco acceso a la atención médica. ${ }^{14}$ También, por la percepción que tienen algunos miembros del personal de salud sobre la incurabilidad del alcoholismo. ${ }^{15}$ Se ha reportado que la percepción que tienen los actores sobre las intervenciones gubernamentales influye en las acciones que ellos efectúan. ${ }^{16}$ Además, a pesar de que se ha mostrado evidencia de la efectividad de las políticas regulatorias de las bebidas

\footnotetext{
* Consumo nocivo de alcohol es un concepto que implica a aquel que tiene efectos sanitarios y sociales perjudiciales en el bebedor, en quienes lo rodean y en la sociedad en general (OMS, 2010:5).
}

alcohólicas, ${ }^{17,18}$ éstas son escasamente cumplidas en relación a la disponibilidad, la asequibilidad ${ }^{19}$ y la restricción de la venta a menores de edad. ${ }^{20}$

\section{OBJETIVO}

Conocer la percepción sobre la manera en como los actores del sistema de salud (políticos, civiles y mercantiles) enfrentan las necesidades de salud derivadas del consumo nocivo de alcohol en una zona rural, con la intención de brindar información para la toma de decisiones en políticas de salud dirigidas a la atención de este problema. Para ello se parte de la observación para describir el escenario local con relación al consumo de alcohol. Asimismo, se identifican las expresiones metafóricas y los dilemas a los que se enfrentan los diferentes actores frente a esta problemática.

\section{MÉTODO}

Investigación cualitativa con diseño de estudio de caso que abarca diferentes unidades de análisis (individuos con diversos papeles sociales), ${ }^{21}$ realizada en una localidad rural de Morelos en la cual sus habitantes identificaron, por medio de un diagnóstico participativo, ${ }^{22}$ que el consumo de bebidas alcohólicas representa un problema de salud en los jóvenes. La localidad es catalogada con un alto grado de marginación y tiene una población de 768 habitantes. ${ }^{11} \mathrm{Su}$ sistema de subsistencia es la agricultura de temporal, corte y venta de palma y madera, emplearse en lugares cercanos o migrar a Estados Unidos; $34 \%$ de los hogares tiene un familiar migrante. ${ }^{22}$

\section{Participantes}

Se utilizó un muestreo teórico que inició con la identificación de voluntarios; continuó con bola de nieve* y se avanzó hacia un muestreo deliberado. Se identificaron cuatro tipos de actores: los pertenecientes al sistema de salud: 1 . jefa de jurisdicción sanitaria, ${ }^{* *}$ médicos, enfermera y auxiliar de salud, 2. sociedad civil: miembros de la agrupación Alcohólicos Anónimos (AA) (facilitadora y miembros de AA), 3. político: funcionarios del gobierno y asuntos públicos (presidente municipal, ayudante municipal, comisariado ejidal, policías) y 4. mercantil: comerciantes de establecimientos locales. Se incluyeron tanto a aquellos comerciantes que venden bebidas alcohólicas como a quienes no lo hacen.

\footnotetext{
* Es una técnica de reclutamiento en la cual se les pide a los participantes de la investigación identifiquen a otros sujetos potencialmente entrevistables, según los criterios de inclusión de la investigación.

* * La jurisdicción sanitaria es una unidad técnico-administrativa desconcentrada por región del Sistema de Salud de los diferentes estados, que cuenta con recursos y facultades para otorgar atención médica a la población no asegurada, con el propósito de conducir adecuadamente las acciones del sector en su área de influencia.
} 
La elección de los cinco prestadores de servicios de salud se basó en los siguientes criterios: experiencia laboral de más de dos años, y ser parte de los servicios de salud de dicha localidad o región sanitaria. En el caso de los actores políticos, debían estar activos en un cargo de elección popular. Para los actores policíacos, civiles y mercantiles, el criterio fue encontrarse trabajando en la localidad.

Se realizó observación participante, entrevistas semiestructuradas y grupos focales. Para cada una de estas técnicas se diseñaron instrumentos con los siguientes ejes temáticos: la percepción de aspectos: a. sociales con relación al consumo de alcohol, b. culturales (costumbres y significados), c. económicos (comercialización), ${ }^{*}$ d. los servicios de salud (acciones de atención y prevención) y e. las políticas que influyen en el consumo de alcohol (regulación, venta y distribución). La aplicación diferenciada de los instrumentos se debió a la información que se quería obtener. Se requería información sobre la manera en cómo cada actor enfrenta esta situación desde sus respectivas funciones, mientras que con los actores civiles nos interesaba la discusión respecto a sus experiencias en su trayectoria como miembros de AA y al consumo de alcohol en su localidad.

\section{Procedimiento}

Se radicó en la localidad de febrero a abril del 2012. Se realizó un acercamiento con las autoridades, líderes y familias de la comunidad y se estableció contacto con diferentes actores involucrados en la venta y consumo de alcohol.

Se hizo mapeo de los comercios de la localidad, identificando el número de tiendas y expendios de alcohol y el tipo de regulación (venta o no a menores de edad, visibilidad del permiso de venta de alcohol, respecto del horario permitido a las tiendas de abarrotes, 22 hrs). Se hizo observación participante en algunos festejos de la comunidad y de la cabecera municipal, como el carnaval. Se acudió a las instalaciones de la unidad móvil itinerante del Programa Oportunidades y de la policía local. El número de entrevistas se estableció de acuerdo con el criterio de saturación teórica. ${ }^{23}$

\section{Análisis de la información}

Se grabaron en audio las entrevistas, los grupos focales y el diario de campo. Se transcribieron las grabaciones y se analizaron con el programa Atlas.ti v.5.

Se tomó como base la aproximación del análisis del discurso $^{24}$ que, por medio de la retórica, permite identificar los argumentos, las metáforas y los dilemas que surgen en la

\footnotetext{
* Los aspectos económicos no solamente se restringen a la comercialización, hay más datos que nos mostrarían un panorama más amplio sobre este rubro como el gasto que se genera para la atención de estas necesidades, la inversión en la compra de las bebidas y el gasto social ocasionado por el ausentismo laboral, incapacidades y falta de participación comunitaria, sin embargo esa carencia de información es una limitación en este trabajo.
}

conversación de los actores en los diversos ámbitos de acción.

La relevancia de analizar las percepciones desde los dilemas y las metáforas expresadas por los actores sociales se debe a que estas últimas juegan un rol central en la cognición, la conducta política y la interacción social, ${ }^{25}$ debido a que facilitan que los problemas complejos tengan sentido, lo cual crea condiciones para que apoyen o resistan ciertas acciones. ${ }^{26,27}$ La metáfora se refiere a la sustitución de una palabra por otra cuyo sentido textual posee cierta semejanza con el sentido literal de la palabra sustituida. ${ }^{28}$

En cambio, los dilemas se generan en el pensamiento que está en constante reflexión de temas opuestos, los cuales surgen entre lo ideológico y el sentido común. Depende de las ventajas y desventajas asociadas a la toma de decisiones a partir de las cuales se efectúan ciertas acciones. El dilema se refiere al argumento formado por la reflexión de temas contrarios que surgen entre lo ideológico y el sentido común. ${ }^{29}$

Se identificaron los fragmentos discursivos que sustentan la argumentación, las metáforas y los temas contrarios, mismos que son enunciados por cada actor en las entrevistas. A su vez, el grupo focal y las notas de campo sirvieron para contextualizar los hallazgos. La información se ordenó en cuatro bloques temáticos: 1. Percepción sobre el consumo de alcohol; 2. percepción sobre la prevención del consumo de alcohol y la atención en salud; 3. percepción sobre la venta de alcohol; 4. percepción sobre la regulación de la venta de alcohol.

El estudio fue aprobado por las comisiones de investigación y ética del Instituto Nacional de Salud Pública. A los participantes se les solicitó consentimiento informado y se les garantizó la confidencialidad.

\section{RESULTADOS}

\section{Escenario local de los actores estudiados}

La comunidad dispone de una Unidad Móvil Itinerante (UMI) del Programa Oportunidades.* El personal de salud está constituido por un médico, una enfermera y una auxiliar (esta última habitante de la localidad). El horario de atención es de las 9:00 a las 14:00 horas, dos días a la semana. La unidad carece de recursos básicos para atender una emergencia de cualquier tipo, incluyendo el tema de la intoxicación etílica. Los horarios representan una barrera de acceso a la atención, además de que gene-

\footnotetext{
* La Unidad Móvil Itinerante forma parte de un programa federal que busca acercar la red de servicios de salud a la población que habita en los municipios con menor índice de desarrollo humano y localidades de alta y muy alta marginación y/o nulo acceso a los servicios de salud. Existen 3 tipos de unidades móviles, pero en el caso que nos ocupa es del tipo I, la cual se encuentra equipada con un consultorio de medicina general, con equipo estándar para la atención primaria, química seca y toma de muestras para cáncer cérvicouterino y equipo de electrocardiografía y está conformado por un médico, un enfermero, un promotor de salud.
} 
Cuadro 1. Población de estudio

\begin{tabular}{|c|c|c|c|c|}
\hline N & Unidad de análisis & Edad & Género & Códigos para identificación \\
\hline 1 & Actores sanitarios & $\begin{array}{l}\bar{X}=39 \text { años } \\
R=(52-30)\end{array}$ & 4 mujeres y 1 hombre & $\begin{array}{l}\text { Jefa de jurisdicción sanitaria: (iis) } \\
\text { Médica: (med m) } \\
\text { Enfermera: (enf) } \\
\text { Auxiliar de salud: (aux) } \\
\text { Médico: (med h) }\end{array}$ \\
\hline 2 & Actores civiles AA & $\begin{array}{l}\bar{X}=45 \text { años } \\
R=(37-57)\end{array}$ & 3 hombres y 1 mujer & $\begin{array}{l}\text { Grupo de apoyo de AA: (G AA) } \\
\text { Facilitadora del grupo de AA: (m AA) }\end{array}$ \\
\hline 3 & Actores políticos y policías & $\begin{array}{l}\bar{X}=43 \text { años } \\
R=(35-57) \\
\bar{X}=38 \text { años } \\
R=(35-40)\end{array}$ & $\begin{array}{l}3 \text { hombres } \\
2 \text { hombres y } 1 \text { mujer }\end{array}$ & $\begin{array}{l}\text { Presidente municipal: (pm) } \\
\text { Ayudante municipal de la localidad: (ayu) } \\
\text { Comisariado ejidal: (com) } \\
\text { Policía 1: (poli 1) } \\
\text { Policía 2: (poli 2) } \\
\text { Policía 3: (poli 3) }\end{array}$ \\
\hline 4 & Actores mercantiles & $\begin{array}{l}\bar{X}=43 \text { años } \\
R=(36-50)\end{array}$ & 5 mujeres & $\begin{array}{l}\text { Comerciante 1: (comer 1) } \\
\text { Comerciante 2: (comer 2) } \\
\text { Comerciante 3: (comer 3) } \\
\text { Comerciante no vende alcohol 1: (comer no 1) } \\
\text { Comerciante no vende alcohol 2: (comer no 2) }\end{array}$ \\
\hline
\end{tabular}

ralmente se da consulta exclusivamente con previa cita. A los médicos los cambian aproximadamente cada año. Los habitantes tienen problemas ante una urgencia médica, ya que el centro de salud más cercano está a 40 minutos en transporte público con un costo que significa el $45 \%$ del salario mínimo diario.

La localidad cuenta con la presencia de un grupo de AA que sesiona dos veces a la semana. La responsable es una mujer, oriunda de la comunidad, quien tiene este cargo desde hace dos años. Es común que las personas de la localidad no acudan al grupo de AA y el grupo sea integrado por personas (seis aproximadamente) de otras poblaciones. Ella menciona que actualmente sólo asisten dos o tres personas de la localidad, de forma esporádica, y desconoce el motivo de esta reducción, ya que anteriormente había más asistentes.

La forma de organización a nivel local es por medio de asambleas mensuales, presididas por la autoridad local (ayudante municipal), donde se toman acuerdos de los diferentes problemas y de las fiestas. La comandancia de policía es un espacio pequeño con una radio para estar en contacto con los guardias municipales; es ocupada por un policía en turnos de 24 horas.

En cuanto al contexto económico-comercial, la falta de opciones de trabajo propicia que parte de la población se dedique al comercio, instalando tiendas de abarrotes en la localidad, en las que además se venden bebidas alcohólicas. En total hay 17 tiendas de abarrotes, de las cuales 11 vendían alcohol. Además, hay viviendas con venta clandestina de alcohol. A excepción de una tienda que mostró su autorización para venta de alcohol, las demás no presentaban los permisos de forma visible. Todas las comerciantes fueron mujeres, propietarias de las tiendas junto con sus familias. Los permisos que brinda el ayuntamiento para la venta de bebidas alcohólicas en tiendas de abarrotes oscilan entre \$4 716 y $\$ 9507$, y se renuevan anualmente al cubrir el costo, que va de $\$ 368.28$ a $\$ 4910$, según la Ley de Ingresos del Municipio $^{30}$ (cuadro 1).

A continuación se muestran las metáforas y dilemas de los cuatro bloques temáticos.

\section{Percepciones acerca del consumo de alcohol}

Existen diferentes percepciones sobre el consumo de alcohol en mujeres por parte de los prestadores de los servicios de salud. La auxiliar de salud y la jefa de jurisdicción sanitaria identifican que el consumo femenino es un problema de salud en la comunidad y en el municipio, respectivamente (necesidad normativa y comparativa). Esta percepción no la comparten los médicos que laboran en la localidad; ellos

Cuadro 2. Dilemas y testimonios sobre el consumo de alcohol

Dilemas Testimonios

Percepciones sobre el ...es un problema de salud pública ....incluconsumo de alcohol de so representa un fenómeno también crecila población femenina do mayormente también en el grupo de la población femenina (iis, p.6)

$$
\text { vs. }
$$

...En cuestión femenino porque no es normal... en la mujer es raro... (med h, p.93)

Dilema: problema vs. si es un problema... con las personas que no representa un pro- no se detienen y no controlan su modo de blema beber... (aux, p.76)

\section{vs.}

...no fue la mayoría... yo no lo tomaría como un problema en esta localidad... (med h, p.60) 
La respuesta social al consumo de alcohol

manifiestan que el consumo es mayormente masculino y que es "raro que la mujer tome" (med h, p.93). Los actores políticos y policíacos identificaron que el consumo de alcohol en mujeres es cada vez más público e incluso es una manera de mostrar "que ya evolucionaron" (poli 1, p.22). Mediante la observación se constató que algunas mujeres menores de edad beben en contextos festivos donde sí es permitido.

La comunidad presenta percepciones contradictorias frente al consumo de alcohol. Los prestadores de servicios de salud tienen diferentes percepciones, pues, por un lado, lo identifican como problema fuerte, y por otro, como una costumbre no problemática en la localidad. Los actores políticos y policíacos lo perciben como una "tradición del pueblo mexicano" (pm, p.55), una costumbre cotidiana en contextos festivos: "se ve mucho borracho en carnaval... es el pan nuestro de cada día" (poli 2, p.321) o según el presidente municipal, como una forma de soportar la precaria situación socio-económica: "Borracho y dormido se me quita lo jodido" (pm, p.14). La mayoría de los comerciantes locales que venden alcohol también identifican que es un problema porque ven a niños consumiéndolo, además de la violencia que ha desatado. Otros comerciantes mencionan que es asunto de pocos. En cambio, las comerciantes que no venden alcohol coincidieron en los conflictos que genera el consumo y afirmaron que por ese motivo no venden alcohol. Estas contradicciones pudieran impedir que el consumo de alcohol se identifique como una necesidad de atención y regulación en salud (cuadro 2).

\section{Percepción sobre la prevención del consumo de alcohol y la atención en salud}

Las autoridades políticas identifican que el consumo de alcohol es un problema de orden público y no de salud. En este sentido, señalan incapacidad para apoyar a las personas que tienen dificultades con el consumo y por tal motivo evitan involucrarse. La forma en que consideran que se puede abordar esta problemática es con apoyar las acciones de prevención. La metáfora usada es "empezar a vacunar (contra el alcohol) en las escuelas" (pm, p.257), aunque no todos creen que se pueda intervenir: "¿pero qué se puede hacer para que dejen de tomar?" (com, p.265).

Tanto los funcionarios públicos de la localidad como los policías reconocen que el consumo de alcohol es una práctica que ellos mismos realizan, y que afecta su salud, pero no se incluyen entre las personas que deben atenderse en los servicios de salud. Ellos optan por acudir a AA, a la iglesia y a jurar;* es la fuerza de voluntad la que les permite salir adelante. La metáfora que permite entender la

\footnotetext{
* La acción de "jurar" es un acto de prometer ante un sacerdote católico no tomar alcohol durante cierto tiempo. Este hecho no fue una referencia constante en los discursos de los actores, sin embargo uno de los actores políticos la practicaba. Otro actor político mencionó que "jurar" era pura farsa, porque sabía que a veces la persona que juró le pedía permiso al sacerdote para que lo dejara tomar.
}

Cuadro 3. Dilemas y testimonios sobre la prevención del consumo de alcohol y la atención en salud

\begin{tabular}{|c|c|}
\hline Dilemas & Testimonios \\
\hline $\begin{array}{l}\text { Involucrarse vs. evitar- } \\
\text { se problemas }\end{array}$ & $\begin{array}{l}\text {...dicen -ayudante métete- y digo -no, } \\
\text { pa'qué si después los problemas son para } \\
\text { mí- (ayu, p.40) }\end{array}$ \\
\hline \multirow{2}{*}{$\begin{array}{l}\text { La atención para dejar } \\
\text { de beber: voluntad vs. } \\
\text { coerción }\end{array}$} & $\begin{array}{l}\text {...para el alcoholismo no hay más que la } \\
\text { fuerza de uno mismo... (poli } 1, \text { p.21) }\end{array}$ \\
\hline & $\begin{array}{l}\text { ¿Qué lugar recomendaría a alguien si tu- } \\
\text { viera problemas con su forma de beber? } \\
\text { (ent*, p.282): hay grupos... pero digo yo } \\
\text { lo anexaría (com, p.283) }\end{array}$ \\
\hline $\begin{array}{l}\text { Curabilidad vs. incura- } \\
\text { bilidad }\end{array}$ & $\begin{array}{l}\text { tratamiento ¿hay? ...el alcoholismo es una } \\
\text { enfermedad social ¿Cómo lo curas? (med } \\
\mathrm{m}, \mathrm{p} .22 \text { ) }\end{array}$ \\
\hline $\begin{array}{l}\text { Priorización del pro- } \\
\text { blema: prioritario vs. } \\
\text { no prioritario }\end{array}$ & $\begin{array}{l}\text {...el alcoholismo es un tema prioritario } \\
\text { pero no el más prioritario... tenemos en } \\
\text { la agenda otros temas que nos preocupan } \\
\text { más por el impacto social que tienen... } \\
\text { o sea yo creo que todavía la población } \\
\text { ¿no?... (ijs, p. 147) }\end{array}$ \\
\hline
\end{tabular}

* Para nombrar a la entrevistadora se utiliza la abreviación ent.

idea de voluntad con una connotación moral es la siguiente: "la cuestión es de nosotros... como en la película de Pedro Infante... que tenían el diablito y el angelito de un lado, generalmente nosotros nos vamos con el diablito" (GAA, p.145). Ellos comparten el ser autoridad y al mismo tiempo el tener la necesidad de salud respecto al consumo de alcohol, pero asumen que la atención para los consumidores excesivos a quienes "el alcohol los bebe a ellos" (ayu, p.44) debe ser mediante la coerción: se necesita "mano dura para la rehabilitación..." (poli 1, p.21).

Los médicos se debaten entre utilidad o ineficacia de las acciones efectuadas para atender a los consumidores de alcohol. Por un lado, perciben la dependencia al alcohol como una enfermedad biológica: "medicamentos para dejar de tomar" (med m, p.5), y por otro opinan que se deben modificar algunas situaciones sociales, como la falta de fuentes de trabajo y la recreación, ante las cuales no pueden influir, lo que expresan con la metáfora: "es una cargota que no nada más es de nosotros" (med m, p.25). Por lo tanto, asumen que es una enfermedad social a la cual no ponen cuidado porque reconocen la limitación que tienen para otorgar una atención adecuada.

La dependencia al alcohol no es un tema prioritario del sector salud, porque existen otras problemáticas más importantes "por el impacto social que tienen, como la mortalidad materna e infantil, el dengue y el alacranismo" (jjs, p.147). Esto conlleva a que, desde la planeación, no sea un tema obligado. Como probable consecuencia de lo anterior, se programan acciones de prevención de manera discontinua. La metáfora usada es: "son cosas al vapor que no funcionan" (med h, p.35). Las actividades de atención también se ven afectadas porque existen prácticas comunes de estigmatización: "tirar de a loco" (al paciente) (med h, p.205), "atenderlos rápido... y 
Cuadro 4. Dilemas y testimonios sobre la venta de alcohol

\begin{tabular}{|c|c|}
\hline Dilemas & Testimonios \\
\hline $\begin{array}{l}\text { Tienda sin ganancia } \\
\text { vs. alcohol como el } \\
\text { gran negocio }\end{array}$ & $\begin{array}{l}\text {...mucha gente piensa que porque vender } \\
\text { alcohol aquí les va a dar más dinero, esa } \\
\text { es su mente de la gente... (comer } 2, \text { p. 19) } \\
\text {...gracias a Dios ...de lo que queda de } \\
\text { ganancia ... aquí comemos... (comer } 2 \text {, } \\
\text { p.142) }\end{array}$ \\
\hline $\begin{array}{l}\text { Festejos: cooperación } \\
\text { para la diversión vs. } \\
\text { problema y lucro }\end{array}$ & $\begin{array}{l}\text {...cuando son bailes organizados por el } \\
\text { ayudante, él mismo compra la bebida, yo } \\
\text { lo veo como tipo negocio para él, porque } \\
\text { la vende... más caro... (aux, p.245) y nos } \\
\text { piden cooperación para la fiesta... (aux, } \\
\text { p.247) }\end{array}$ \\
\hline
\end{tabular}

vámonos" (mandarlos a casa) (med h, p.213), y asumir que la atención a estos pacientes "es una pérdida de dinero que se debería utilizar para otras cosas" (med m, p.27). Referir al segundo nivel de atención no es una opción, ya que los médicos desconfían de su utilidad debido a la escasez de este tipo de centros y de la capacidad para resolver con eficacia dicha problemática (cuadro 3).

\section{Percepción de la venta de alcohol}

La venta de alcohol es percibida por la población como un negocio, tanto para el comerciante como para el ayuntamiento. La autoridad puede lucrar con los permisos otorgados para la venta: "es que realmente no les conviene a ellos (regular la venta), porque te cobran un billetote" (por los permisos) (poli 2, p.133-139).

Se tiene la percepción de que una tienda, si no vende alcohol o cigarros, no tiene el suficiente ingreso para obtener ganancia. Las autoridades consideran que la generación de la diversión se da únicamente por el consumo de alcohol en

Cuadro 5. Dilemas y testimonios sobre la regulación de la venta de alcohol

\begin{tabular}{|c|c|}
\hline Dilemas & Testimonios \\
\hline $\begin{array}{l}\text { Reglamentable vs. irre- } \\
\text { glamentable }\end{array}$ & $\begin{array}{l}\text {...Si buscásemos la forma de restringir } \\
\text { pues ya vio lo que pasó en Estados Unidos } \\
\text { en } 1920, \ldots \text { que hubo venta de alcohol in- } \\
\text { discriminada clandestinamente (pm, p.98) }\end{array}$ \\
\hline $\begin{array}{l}\text { Difícil aplicación de la } \\
\text { regulación vs. confu- } \\
\text { sión de deberes }\end{array}$ & $\begin{array}{l}\text {...nunca serían suficientes tantos... verifica- } \\
\text { dores que pudieran estar pendientes de... } \\
\text { venta de alcohol (a menores) ... hay cosas } \\
\text { que están en la ley pero es muy difícil su } \\
\text { aplicación... (ijs, p. 18) }\end{array}$ \\
\hline $\begin{array}{l}\text { Beneficios económi- } \\
\text { cos: por la aplicación } \\
\text { del reglamento vs. por } \\
\text { negligencia }\end{array}$ & $\begin{array}{l}\text {...ellos tienen un permiso... porque pagan } \\
\text { horas extras y es un ingreso para el munici- } \\
\text { pio... (pm, p. 140) }\end{array}$ \\
\hline $\begin{array}{l}\text { Todos infringimos el } \\
\text { reglamento vs. nadie } \\
\text { denuncia }\end{array}$ & $\begin{array}{l}\text {... la misma gente no lo ha reportado... } \\
\text { pero el día que lo haga pues ni modo... } \\
\text { tendría que proceder... pero si la gente no } \\
\text { dice nada... (ayu, p. 143) }\end{array}$ \\
\hline
\end{tabular}

las fiestas, sin embargo, critican los problemas de violencia y accidentes ocasionados por éste. Ellos piden cooperación para el festejo, pero esa colaboración después es percibida por los prestadores de servicios de salud y civiles como negocio de las autoridades en relación con la venta de bebidas (cuadro 4).

\section{Percepción sobre la regulación de la venta de alcohol}

Este apartado agrupa argumentos que favorecen la reglamentación de la venta y contrasta con las percepciones que cuestionan la utilidad y la viabilidad de las restricciones debido a la corrupción: "que ahi te va una feria y yo me voy a continuar" (poli 2, p.373) (cuadro 5). Se afirma que sólo es posible controlar la venta de bebidas alcohólicas a menores de edad y la aplicación de los horarios, a excepción de las épocas festivas, ya que: "el carnaval es una cantina gigante... donde se da rienda suelta a los instintos humanos" (pm, p.46) y "todo se vale en el carnaval" (poli 2, p.271).

Los funcionarios del gobierno local temen aplicar la regulación porque consideran que se incrementaría la venta clandestina. Incluso piensan que no se puede hacer nada que impida que la gente lo consuma.

Se pone de manifiesto la dificultad que perciben los actores políticos para aplicar el reglamento, lo cual contrasta con la confusión de deberes en la aplicación del mismo. Ellos se justifican señalando que, cuando se intenta obligar a respetar los horarios de venta, los comerciantes se molestan: "luego la gente se te va encima" (com, p.189). Los policías muestran desconocimiento de la reglamentación al justificar la falta de vigilancia de la regulación y culpar a los padres y madres de familia por ser quienes facilitan el alcohol a sus hijos e hijas. Al respecto, el artículo 201 del Código Penal Federal menciona: "Comete el delito de corrupción, quien obligue, induzca, facilite o procure a una o varias personas menores de edad el consumo habitual de bebidas alcohólicas". ${ }^{31}$ En el registro etnográfico, durante el carnaval, se observó que las bebidas alcohólicas se vendían sin ninguna restricción a menores de edad o uniformados, sin que ninguna autoridad supervisara o restringiera la venta. $\mathrm{Al}$ parecer, todos saben que se debe vigilar la regulación, pero no tienen claro a quién le corresponde.

Uno de los beneficios económicos en los municipios del país, si se aplican los reglamentos, es el incentivo de Pueblo Mágico, ${ }^{*, 32,33}$ con el cual se comprometen a vigilar el consumo alcohol en la vía pública. Además, se pueden obtener

\footnotetext{
* El Programa Pueblos Mágicos contribuye a revalorar a un conjunto de poblaciones del país que siempre han estado en el imaginario colectivo de la nación en su conjunto, y que representan alternativas frescas y diferentes para los visitantes nacionales y extranjeros. Más que un rescate, es un reconocimiento a quienes habitan esos hermosos lugares de la geografía mexicana y han sabido guardar para todos la riqueza cultural e histórica que encierran. ${ }^{31}$ Específicamente en Tepoztlán, para preservar la denominación de Pueblo Mágico y seguir obteniendo los recursos que destina el Gobierno para su conservación se implementó en el carnaval del 2013 el control de la venta de alcohol. ${ }^{32}$
} 
otros ingresos por aplicar el alcoholímetro. En sentido contrario, está la obtención de recursos al otorgar los permisos de venta de bebidas alcohólicas. Existen acuerdos, que varían en cada municipio, y que permiten o prohiben la venta nocturna de alcohol. Las metáforas que expresan este tipo de ganancia para el municipio del estudio son las siguientes: "es una mina de oro" (poli 2, p.381) y "es el agosto de todos los ayuntamientos" (poli 2, p.42).

Este último dilema expone la complicidad existente entre actores políticos y mercantiles, quienes infringen la prohibición de la venta a menores de edad y la regulación de los horarios, pero a la vez los actores políticos se excusan de no hacer nada mientras no haya denuncia por parte de la población. Esta percepción sobre la imposibilidad de regular los horarios de venta de las bebidas alcohólicas resalta la vulnerabilidad del Estado ante los intereses mercantiles en el nivel local. Esta "complicidad antirregulatoria"** por parte de diversos actores se puede apreciar en distintos niveles.

\section{DISCUSIÓN Y CONCLUSIÓN}

En el presente estudio se profundizó en la comprensión de la forma en que diversos actores sociales enfrentan las necesidades de salud derivadas del consumo nocivo de alcohol a nivel local. El tipo de análisis fue útil para conocer cómo una serie de problemas complejos cobran sentido para los diferentes actores e influyen en su conducta y su interacción social favoreciendo ciertas prácticas sociales.

Respecto a la percepción sobre las necesidades de salud, si bien el consumo de alcohol fue un problema expresado por los miembros de la comunidad, se identificó que existen diferencias sobre la valoración que hacen los diferentes actores. Estas discrepancias pudieran deberse a motivos como la permanencia en el lugar, la capacitación y la experiencia sobre el tema. Se encontró que la respuesta a las necesidades de atención, prevención y regulación en este contexto, es compleja, porque, por un lado, emergieron metáforas como que las mujeres ya "evolucionaron" por consumir alcohol, lo cual pudiera entorpecer las acciones de prevención y atención a este grupo poblacional. Por otro lado, los actores sociales, responsables de la planeación de acciones en salud a nivel local (jefes de jurisdicción sanitaria en coordinación con los funcionarios de gobierno), omiten tomar en cuenta que el consumo de alcohol es valorado de distintas formas y se efectúan acciones de regulación y atención de limitado alcance para disminuir el consumo nocivo de alcohol.

El análisis del discurso, por medio de las metáforas y de los dilemas, permitió identificar cómo las prácticas dis-

\footnotetext{
* La complicidad antirregulatoria es una categoría creada en este trabajo, y se refiere a la interiorización de un conjunto de relaciones entre los actores del Estado y del mercado, los cuales justifican la inacción de los actores ante los conflictos generados entre las ganancias de la disponibilidad de bebidas en el mercado y su regulación en pro del bienestar colectivo.
}

cursivas de los actores mantienen y promueven ciertas relaciones sociales $^{22}$ en torno al consumo de alcohol. Algunas de ellas se mencionan a continuación: a. la falta de aplicación de vigilancia entre los actores encargados de la regulación de la venta, al percibir que el consumo es una "tradición del pueblo mexicano"; b. la escasa o nula demanda de atención que reportan los médicos para atender estos problemas, que pudiera deberse a la percepción de que es un problema que se resuelve de manera personal con voluntad y buen comportamiento y c. la arbitrariedad para vender bebidas alcohólicas a menores de edad debido a que, en contextos festivos, "todo se vale" y por lo tanto se debe aprovechar "la mina de oro", tanto en el otorgamiento de permisos como en la venta de bebidas embriagantes.

Se observa una inercia en cuanto a la falta de acciones por parte de los prestadores de servicios de salud y funcionarios de gobierno locales, quienes perciben el consumo de alcohol como un problema cultural inmodificable, como lo ha descrito Menéndez, y lo vuelve banal y opaco; ${ }^{34}$ por consiguiente, lo justifican. Esta pasividad puede estar favorecida por percepciones prejuiciosas y falta de asignación de recursos para capacitación del personal de salud en esta temática, lo cual lleva a que no se atienda o no se haga la referencia a las unidades médicas especializadas en adicciones.

En el ámbito de la atención a la salud por parte de AA, las metáforas utilizadas influyen en el tipo de respuesta que se da, por ejemplo, "nosotros nos vamos con el diablito". El sentido de esta frase tiene una connotación moral y, por tanto, el problema se reduce a que la persona lo enfrente con voluntad, invisibilizando las causas estructurales, como la falta de oportunidades de recreación, estudio y trabajo para los habitantes, así como la limitada aplicación de regulación en la venta, distribución y publicidad de bebidas alcohólicas, lo cual beneficia la producción de estos productos. Algo semejante sucede con enfermedades que tienen un estigma moralizante, como la obesidad ${ }^{35}$ y el VIH-SIDA, ${ }^{36}$ en las cuales se han reportado metáforas que "culpabilizan" al individuo de su situación y no toman en consideración las causas estructurales.

Si el consumo de alcohol es percibido como un problema de voluntad, se asume que no se requiere ningún tipo de restricción, regulación o atención, porque todo depende de una decisión personal. Haciendo un paralelismo con la obesidad, esto concuerda con los resultados de Oliver y Lee, ${ }^{16}$ quienes señalan que los individuos entrevistados que atribuyen la obesidad a las elecciones personales mostraron dificultades para reconocer el papel que juega el Estado en el comportamiento individual. Se ha documentado que una de las consecuencias de la culpabilización de la persona por su modo de vida, es desenfocar el panorama de los determinantes estructurales que favorecen estas situaciones de consumo excesivo y que limitan el planteamiento de alternativas de solución. ${ }^{37}$

Las metáforas respecto a la prevención, como "vacunar en las escuelas", muestran la dificultad que tienen algunos 
prestadores de servicios de salud y funcionarios del gobierno local para concebir la atención y prevención fuera del campo biológico. Esto concuerda con las limitaciones de la práctica médica basada en un enfoque más curativo que de prevención y promoción de la salud, bajo un modelo médico biologizado, ahistórico y asocial conocido como el Modelo Médico Hegemónico. ${ }^{38}$ Esta interpretación biológica de una problemática social, como el consumo de bebidas alcohólicas, coincide con señalamientos que otras autoras han hecho para fenómenos como la violencia en familia. ${ }^{39}$

La metáfora "tirar de a loco" muestra las actitudes discriminatorias de algunos médicos para atender a personas bajo los efectos del alcohol, que van desde estigmatizarlas hasta hacerlos sentir que son una "cargota" para el sector salud. Lo anterior, es semejante a lo reportado por Mondragón et al. ${ }^{40}$ quienes mencionan actitudes de disgusto y rechazo por parte del personal de salud hacia los pacientes que llegan alcoholizados, y también en los tratos diferenciales a las personas con SIDA. ${ }^{41}$

La identidad masculina tiene como una de sus características el asumir riesgos. Además, se "naturaliza" el que los hombres consuman alcohol. Esta situación se asume como un aspecto cultural que no se puede cambiar; es decir, no es percibida como una necesidad de atención en salud. En tanto, el consumo femenino aún se debate entre ser concebido como un problema de salud pública o un asunto invisibilizado. Lo anterior resalta la necesaria inclusión de programas con perspectiva de género en la atención al consumo de alcohol, tal como lo han reportado otros autores. ${ }^{42}$

Los dilemas en la venta de alcohol muestran cómo los determinantes socioeconómicos propician situaciones en las que las personas, por escasez de fuentes de trabajo, deciden vender bebidas alcohólicas como un medio de subsistencia. Al respecto, Boltvinik ${ }^{43}$ menciona que para estudiar los determinantes de las necesidades se debe recurrir al análisis de las condiciones de la reproducción de la fuerza de trabajo, el proceso de producción de consumo y la venta.

Las metáforas relacionadas con la regulación de la venta y distribución de bebidas alcohólicas expresan el descontrol y la dificultad que se genera para su aplicación. La falta de regulación y las condiciones económicas precarias han llevado a que, en los últimos tres lustros, se incrementen la distribución y la asequibilidad de bebidas alcohólicas, entre otros productos que se han identificado como nocivos para la salud. Lo anterior coincide con el incremento de consumo de estos productos en los países de bajo y mediano ingreso. ${ }^{44}$

Este estudio brinda una mirada para comprender las respuestas contradictorias y evasivas a las necesidades de salud que se reflejan mediante el análisis del discurso de los actores involucrados. También permitió conocer cómo la experiencia personal se mezcla o se contradice con los deberes del cargo de los actores sociales, lo cual influye para ejercer su práctica y justifica en ocasiones la desatención del problema.

Es necesario generar una investigación que apoye al sistema de salud a resolver los dilemas surgidos en la atención y en la regulación del consumo de alcohol. Generar este tipo de información por medio de estudios de caso, metodología cualitativa y análisis del discurso, resalta la importancia de las iniciativas de promoción de la salud a nivel local. ${ }^{45-47}$

Por lo tanto, coincidimos con lo que proponen Campos y Mishima, ${ }^{48}$ acerca de que la respuesta del Sistema de Salud a las necesidades de la población tiene que ser intersectorial y favorecer la presencia del Estado para garantizar los servicios que promueven el bienestar y la participación social. Es decir, una práctica que supere el ámbito curativo, que incida en los determinantes, en la salud como derecho universal y en el mejoramiento de la condición humana.

Una limitación del estudio es su duración en el trabajo de campo que limitó la incorporación de más actores y momentos de observación. Así también, es posible que la gente del lugar se comportara diferente a como lo venían haciendo debido a la presencia de la investigadora. Por tal motivo, puede haber sesgos en el proceso de producción, obtención y análisis de los datos. Sin embargo, la variedad de fuentes e informantes y de técnicas de recolección de datos e investigadores intentó aminorarlo.

Para diseñar una política pública que responda a las necesidades de salud relacionada al consumo de alcohol se debe, por un lado, tomar en cuenta la naturaleza dilemática del pensamiento social de los individuos que intervienen en dicha respuesta.

Se sugiere que la política pública fortalezca cuatro áreas: a. atención primaria para zonas rurales; favorecer la permanencia de los prestadores de servicios de salud, debido a que la constante rotación hace que se desconozcan las problemáticas locales, además de establecer otros mecanismos de acercamiento como el monitoreo, las encuestas de opinión, de solicitud de servicios, entre otras; b. en la promoción de la salud, la incorporación de promotores que ejerzan funciones de abogacía para superar resistencias, como difundir los procesos de AA, informar sobre el consumo nocivo de alcohol e incorporar un enfoque preventivo con perspectiva de género. Es importante la identificación de los actores con los cuales se puede generar sinergia para brindar una respuesta integral mediante la formación de comisiones locales contra las adicciones; c. en el caso de la regulación se requiere precisión para la aplicación y el cumplimiento de la misma, además de fomentar mayor participación como ciudadanos para efectuar y vigilar la normatividad. Esto se puede lograr incorporando a los actores sociales desde el diseño y operación de las políticas y programas con un ejercicio permanente de evaluación; d. incidir en determinantes sociales, tales como aumentar las fuentes de trabajo, recreación, educación y acceso a los servicios de salud. 


\section{Financiamiento}

Ninguno.

\section{Conflicto de intereses}

Los autores declararon no tener conflicto de intereses.

\section{REFERENCIAS}

1. Organización Mundial de la Salud. Estrategia mundial para reducir el uso nocivo de alcohol. Suiza: 2010.

2. Bava S, Tapert SF. Adolescent brain development and the risk for alcohol and other drug problems. Neuropsychology Review 2010;20:398-413.

3. Silveri MM. Adolescent brain development and underage drinking in the United States: identifying risks of alcohol use in college populations. Harv Rev of psychiatry 2012;20:189-200.

4. Wetherill R, Tapert, SF. Adolescent Brain Development, Substance Use, and Psychotherapeutic Change. Psychol Addict Behav 2012.

5. Cavanagh S, Chadwick K. Health needs assessment: a practical guide. En: National Institute for health and Clinical Excellence 2005.Disponible en: http://www.nice.org.uk/media/150/35/health_needs_assessment_a_practical_guide.pdf. Consultado el 10 de marzo de 2012.

6. Rawaf S, Bahl V. Assesing health needs of people from Minority Ethnic Groups. Londres: Royal College of Physicians of London; 1998.

7. Secretaría de Salud, Consejo Nacional Contra las Adicciones, Instituto Nacional de Psiquiatría Ramón de la Fuente, Instituto Nacional de Salud Pública. Publicación especial. Encuesta nacional de adicciones 2008. Reporte de resultados nacionales. México: 2009.

8. Instituto Nacional de Psiquiatría Ramón de la Fuente Muñiz; Instituto Nacional de Salud Publica; Secretaría de Salud. Encuesta nacional de adicciones 2011: Reporte de alcohol. Medina-Mora ME, Villatoro-Velazquez JA, Fleiz-Bautista C, Téllez-Rojo MM et al. México DF: 2012. Disponible en: www.inprf.gob.mx. Consultado el 10 de mayo de 2013.

9. Medina-Mora ME, Villatoro J, Juárez F. Underage drinking in México. Trabajo presentado en Kettil Bruun Society Conference on Alcohol. Toronto: Manuscrito interno; 2002.

10. Medina-Mora ME. Mexicans and alcohol: patterns, problems and policies. Addiction 2007;102:1041-1045.

11. Consejo Nacional de Población 2010. Índice de marginación por localidad. Disponible en: http://www.conapo.gob.mx/es/CONAPO/Indice_ de_Marginacion_por_Localidad_2010. Consultado el 3 de enero de 2013.

12. Secretaría de Salud, Consejo Nacional Contra las Adicciones. Encuesta nacional de adicciones Tabaco, alcohol y otras drogas: resumen ejecutivo. México: 2002.

13. Pozas Arciniega R. El alcoholismo y la organización social, 1957 En: Menéndez EL (ed.). Antropología del alcoholismo en México: los límites culturales de la economía política (1930-1979). Ciesas 361991.

14. Natera Rey G, Medina Aguilar PS, Callejas Pérez F, Juárez F et al. Efectos de una intervención a familiares de consumidores de alcohol en una región indígena en México. Salud Mental 2011;34:195-201.

15. Menéndez E, Di Pardo RD. De algunos alcoholismos y algunos saberes. Atención primaria y proceso de alcoholización. México: Centro de investigaciones y Estudios superiores en Antropología social; 1996.

16. Oliver JE, Lee T. Public opinion and the politics of obesity in America. J Health Politics, Policy Law 2005;30:923-954.

17. Organización Panamericana de la Salud. El alcohol: un producto de consumo no ordinario. Investigación y políticas públicas. Segunda ed. Washington DC: OPS; 2010.

18. Medina-Mora ME, García-Téllez I, Cortina D, Orozco R et al. Estudio de costo-efectividad de intervenciones para prevenir el abuso de alcohol en México. Salud Mental 2010;33:373-378.

19. Stuckler D, McKee M, Ebrahim S, Basu S. Manufacturing epidemics: The role of global producers in increased consumption of unhealthy commodities including processed foods, alcohol, and tobacco. PLoS Med 2012;9:1-8.

20. Secretaría de Salud. Cruzada nacional contra el consumo de alcohol entre adolescentes. México, DF: 2010 Disponible en: http://www.jornada. unam.mx/2013/04/02/politica/013n1pol. Consultado el 1 de abril de 2013.

21. Gundermann Kröll H. El método de los estudios de caso, en Tarrés ML (comp.) Observar, escuchar y comprender sobre la tradición cualitativa en la investigación social. México: Colegio de México, FLACSO; 2008.

22. Arenas-Monreal L, Cortez-Lugo M, Parada-Toro I. Community-based participatory research and the Escuela de Salud Publica in Mexico. Public Health Rep 2011;126:436-440.

23. Glasser BG, Strauss AL. The discovery of grounded theory: Strategies for qualitative research. USA: Aldine de Gruyter; 1967.

24. Íñiguez L, Antaki C. El análisis del discurso en psicología social. Boletín de Psicología 1994;44:63.

25. Lakoff G, Johnson M. Metaphors we live by. Chicago: University of Chicago Press; 1980.

26. Schlesinger $M$, Lau RR. The meaning and measure of policy metaphors. American Political Science Review 2000;94:611-626.

27. Barry CL, Brescoll VL, Brownell KD, Schlesinger M. Obesity metaphors: how beliefs about the causes of obesity affect support for public policy. USA: Milbank Quarterly 2009; Vol.87 pp.7-47.

28. Mortará Garavelli B. Manual de retórica. Madrid: Cátedra; 1988.

29. Billig M, Condor S, Edwards D, Gane M et al. Ideological dilemmas. A social psychology of everyday thinking. Londres: Sage Publications; 1988.

30. Gobierno del Estado de Morelos. Ley de ingresos del Municipio de Yautepec, Mor, para el ejercicio fiscal del año 2012. Disponible en: http:// www.morelos.gob.mx/10consejeria/files/Leyes $\% 20 \mathrm{de} \% 20$ Ingresos $\% 2020$ 12/Ley-Ingresos-Yautepec2012.pdf. Consultado el 12 de febrero de 2012.

31. Cámara de Diputados del H. Congreso de la Unión. Código penal federal. Última reforma DOF 07-06-2013. Disponible en: http://www.diputados.gob.mx/LeyesBiblio/pdf/9.pdf. Consultado el 10 de junio de 2013.

32. Secretaría de Turismo. Pueblos mágicos, reglas de operación, México. Disponible en: http://www.sectur.gob.mx/work/models/sectur/ Resource/99fbd793-a344-4b98-9633-78607f33cb8f/Reglas_de_operacion.pdf. Consultado el 10 de junio de 2013.

33. Diario de Morelos. Controla Tépoz la venta de alcohol. Artículo del 11 de febrero de 2013. Disponible en: http://www.diariodemorelos.com/article/ controla-tépoz-la-venta-de-alcohol. Consultado el 23 de febrero de 2013.

34. Menéndez E, Di Pardo R. Alcoholismo: políticas e incongruencias del sector salud en México. Desacatos 2006;20:29-52.

35. Schlesinger M, Brescoll VL, Barry CB. Constructing policy metaphors for obesity. Working paper, Yale Rudd Center for Food Policy and Obesity. 2008 En: Barry CL, Brescoll VL, Brownell KD, Schlesinger M (ed). Obesity metaphors: how beliefs about the causes of obesity affect support for public policy. USA Milbank Quarterly 2009;87:7-47.

36. Sontag S. La enfermedad y sus metáforas y el SIDA y sus metáforas. Madrid: Taurus; 1996.

37. Alfonso OR, Lladó AP. Jóvenes y riesgos ¿Unas relaciones ineludibles Barcelona: Ed. Bellaterra; 2010.

38. Menéndez E. Morir de alcohol: Saber y hegemonía médica. 1ra ed. México: Consejo Nacional para la Cultura y las Artes, Alianza Editorial Mexicana; 1990.

39. Herrera C, Agoff C. Dilemas del personal médico ante la violencia de pareja en México. Cad Saúde Pública 2006;22:2349-2357.

40. Mondragón BL, Romero M, Borges G. Etnography in an emergency room: Evaluating patients with alcohol consumption. Salud Pública México 2008;50:308-315.

41. Infante $\mathrm{C}$, Zarco A, Cuadra SM, Morrison $\mathrm{K}$ et al. El estigma asociado al VIH/SIDA: el caso de los prestadores de servicios de salud en México. Salud Pública México 2006;48:141-150.

42. Belló M, Puentes-Rosas E, Medina-Mora ME. El papel del género en la demanda de atención por problemas asociados al consumo de alcohol en México. Rev Panam Salud Pública 2008;23:231-236.

43. Boltvinik J. Pobreza y necesidades básicas: conceptos y métodos de 
medición. Caracas: PNUD Proyecto Regional para la Superación de la Pobreza; 1990.

44. Stuckler D, Nestle M. Big food, food systems, and global health. PLoS Med 2012;9:4.

45. Organización Panamericana de la Salud, Organización Mundial de la Salud. Los proyectos locales de promoción de la salud. Nuevos escenarios para el desarrollo de la salud pública. Cuba: OPS, OMS; 1995.

46. Álvarez Pérez, AG, García Fariñas A, Rodríguez Salvá A, Bonet Gor- bea M. Voluntad política y acción intersectorial: Premisas clave para la determinación social de la salud en Cuba. Revista Cubana Higiene Epidemiología 2007;45:1-15.

47. Arenas Monreal ML, Sosa Delgado N, Corrales Trujillo A (Coord). Experiencia de acercamiento comunitario y participación de la población en salud. Morelos: SEDESOL, INDESOL, INSP; 2008.

48. Campos CMS, Mishima SM. Necessidades de saúde pela voz da sociedade civil e do Estado. Cad Saude Pública 2005;21:1260-1268. 